\title{
COMPARISON OF COMPLETE DECONGESTIVE THERAPY AND KINESIOLOGY TAPING FOR UNILATERAL UPPER LIMB BREAST CANCER-RELATED LYMPHEDEMA: A RANDOMIZED CONTROLLED TRIAL
}

\author{
C. Basoglu, D. Sindel, M. Corum, A. Oral
}

Department of Physical Medicine and Rehabilitation (CB,DS,AO), Istanbul Faculty of Medicine, Istanbul University, and Department of Physical Medicine and Rehabilitation (MC), Istanbul Physical Medicine and Rehabilitation Training and Research Hospital, Istanbul, Turkey

\section{ABSTRACT}

We designed a study to compare effects of complete decongestive therapy (CDT) and kinesiology taping (KT) (with exercise and skin care) on limb circumference, lymphedema volume, grip strength, functional status, and quality of life in patients with unilateral breast cancer-related lymphedema (BCRL). Forty patients with unilateral stage $2 B C R L$ were randomized to either the CDT group $(n=20)$ or the KT group $(n=20)$. Patients in the CDT group underwent 30-min manual lymphatic drainage (MLD) and multi-layer, short-stretch bandaging once a week for four weeks. Patients in the KT group underwent taping once a week for four weeks. In addition, all patients were informed about skin care and given an exercise program throughout the treatment. Upper extremity circumference and volume differences as primary outcomes and grip strength, Quick-Disabilities of the Arm, Shoulder and Hand (Q-DASH), and Functional Assessment of Cancer Therapy-Breast (FACT-B) scores as secondary outcomes were assessed initially, after treatment (4 weeks), and at the 1st month follow-up. Limb circumference and volume differences were significantly reduced in the CDT group after the 4-week treatment compared with the KT group ( $p=0.012$ and $p=0.015$, respectively), but there was no difference between the groups in the 1st month follow-up ( $p>0.05)$. There was no difference between the groups in terms of grip strength, $Q-D A S H$, and FACT-B scores after treatment and at the 1st month follow-up ( $p>0.05)$. Our results show that both KT and CDT were found to significantly reduce limb volume and circumference individually at 4-weeks and the one-month follow-up in patients with BCRL and that CDT significantly reduced both limb volume and circumference compared to KT at the 4week time point, but not at the follow-up. Further randomized controlled trials with patients at different stages of BCRL are needed to confirm and expand these results.

Keywords: breast cancer lymphedema, kinesiotape treatment, CDT (complete decongestive therapy) treatment, Q-DASH, FACT-B, randomized controlled trial

Recent developments in early detection methods and new treatment modalities for breast cancer have improved the 5-year survival rates to $90 \%$ (1). As the duration of survival increased, physicians have focused more attention on treatment-related chronic 
sequelae such as lymphedema (2). Lymphedema is a condition characterized by the accumulation of protein-rich fluid in the interstitial space secondary to inadequacy of the lymphatic system $(3,4)$. Breast cancerrelated lymphedema (BCRL) is usually caused by disruption of lymphatic drainage of the upper extremity as a result of axillary lymph node dissection and/or axillary radiation (5). Its incidence has been reported as $16.6 \%$ (6). Problems such as pain, heaviness, tension, and decreased range of motion (ROM) associated with BCRL cause impairment in upper limb function and decrease quality of life, and therefore require long-term, lifelong treatment $(7,8)$.

Complete decongestive therapy (CDT) consists of manual lymphatic drainage (MLD), compression bandages, exercise, and skin care. Compression with garments and pneumatic pumps), MLD, and exercise (resistive, aerobic, acoustic) have also been utilized as non-invasive treatment approaches for the management of lymphedema (9-11). CDT is recognized by the International Society of Lymphology (ISL) as the current international standard treatment approach for lymphedema (12) to reduce edema and sustain reduction over the long term. Although some systematic reviews reported that CDT was an effective treatment method for reducing arm volume in $\operatorname{BRCL}(9,13)$, a recent systematic review by Jeffs et al. (14) on the clinical efficacy of CDT on arm volume, function, and quality of life showed poor evidence in women with early lymphedema (duration of BCRL symptoms less than 12 months).

Kinesiology taping (KT) with a lymphatic correction technique is used in order to support lymphatic circulation (15) as an adjuncttive treatment for lymphedema. KT is designed to reduce pressure on tissue-level lymphatics and create a space that allows circulation in the tissue (16). It is also thought to have effect on pain by increasing the stimulation of the mechanoreceptors by providing proprioceptive feedback through the skin (17). In previous systematic reviews and meta- analyses, KT was found to be effective in BCRL but not superior to other treatments $(18,19)$.

In this study, we aimed to compare the effectiveness of CDT to KT (with skin care and exercise) in the treatment of female patients with BCRL in an effort to provide a more comfortable, less expensive, and noninvasive alternative treatment.

\section{PATIENTS AND METHODS}

Forty patients with grade 2 lymphedema, who were admitted between September 2014 and March 2015, were randomized equally with an allocation rate of $1: 1$ by using a computer program into two groups: CDT $(n=20)$ including compression bandage, MLD, skin care, and therapeutic exercises and KT $(n=20)$, including skin care and exercise, and no MLD or bandaging. The study is prospective, randomized, and controlled with one month follow-up.

Written and oral information regarding purpose, duration, and treatment methods were provided to participants and written informed consent was obtained from all participants. The Research Ethics Committee of Istanbul University approved the study protocol, which was conducted in accordance with the principles of the Helsinki Declaration (IRB Study protocol: 2015/369).

Women were enrolled in the study if they were over 18 years, had unilateral grade 2 BCRL of at least 6 months after breast cancer treatment, their radiotherapy treatment ended at least 2 months prior, and who had a difference of more than $2 \mathrm{~cm}$ between the two arms in terms of arm circumference measurements.

Patients were excluded if they had stage 1 and 3 lymphedema, active cancer, skin infection, radiation burns, severely affected upper extremity ROM, structural disorders of the upper limbs, kidney failure, heart failure, a history of untreated deep venous thrombosis, and a body mass index (BMI) $\geq 35 \mathrm{~kg} / \mathrm{m}^{2}$. In addition, patients who had received a CDT program within the last 3 months and those 
with a bandage allergy were also excluded from the study.

Patients were treated 5 days per week and not given treatment for 2 days during the weekends. All patients were informed about skin care and were asked to perform a 20minute exercise (upper limb ROM exercises, pumping, upper limb stretching, and isometric strengthening exercises) until the $1^{\text {st }}$ month follow-up.

\section{Complete Decongestive Therapy}

The standard CDT group was treated by an experienced physiotherapist for the first week, 5 days a week. MLD was performed following the training of the Földi method. Prior to bandaging, 30 minutes of MLD was applied by working from the affected limb towards the unaffected side (to anterior trunk, posterior trunk, and affected limb) with the same standardized protocol. MLD was applied to the side of the affected limb quadrant, starting at the base of the neck and then progressing to the limb. The affected limb was always treated proximally from the upper arm to the axilla, and then from the hand to the elbow. Finally, the whole limb was massaged from the distal to the proximal. Multi-layer, shortstretch bandages were used for compression. During the first week of practice, the patients and the patient's relatives were given practical training on bandaging and MLD in each session. During the other weeks, patients continued CDT with self-bandaging and MLD, so that the duration of treatment was 4 weeks. All patients were asked to keep the multilayer bandage on for at least 20-22 hours a day.

\section{Kinesiology Taping}

In the KT group, 5-cm wide standard tapes (Kinesio ${ }^{\circledR}$ Tex Classic) were used in all applications without color preference. KT was performed by a physician once a week for four weeks for a total of four sessions. A total of 6 tapes, 3 above the elbow and 3 below the elbow, were applied on the affected extremity

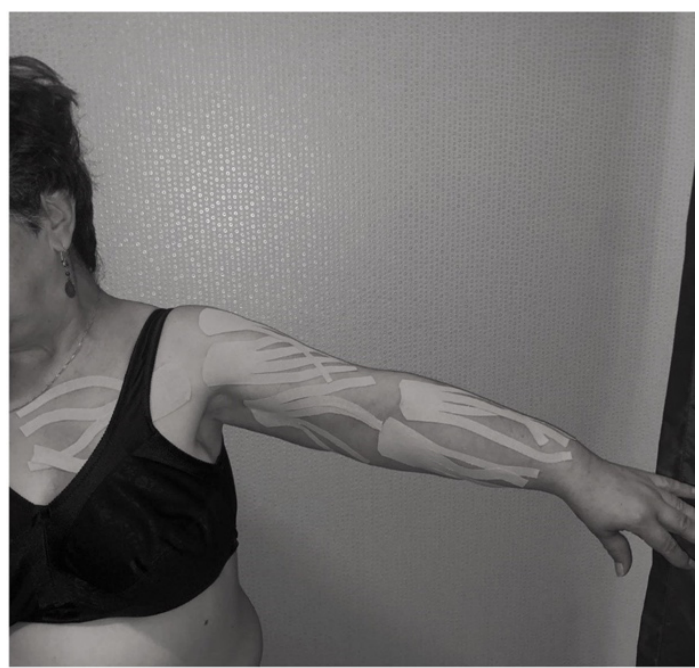

Fig. 1. Example of a patient with kinesiology taping pattern utilized in this study applied to upper limb.

with the lymphatic correction method in the KT group. The tapes were applied in spiral and cross shapes along the arm, forearm and anterior axillary-axillary anastomoses on the affected extremity. The length of the kinesiology tape was adjusted according to the patients' limb length. The tapes were prepared to be 4-lane (4 strip fan cut), and the $5 \mathrm{~cm}$ section at the end was left uncut. The uncut (anchor) end of the tape was placed near the unaffected lymph node and in accordance with the direction of the lymphatic flow. No stretching was applied to the uncut end and strip sections. A further tape was also applied towards the unaffected axillary area at the front of the chest. It was applied so that the ribbon sections cross each other (Fig. 1). If the tape was removed for any reason before the desired time, we examined the patient and the tape was renewed.

\section{Outcomes}

The results were evaluated at baseline, after four weeks of treatment, and at one month of follow-up. All measurements were obtained by an assessor blinded to the treatments. In addition, upper limb circumference and volume differences were recorded weekly 
until the end of treatment.

\section{Primary outcomes: upper extremity circumference and volume differences}

Limb circumference measurements were performed at four points, the metacarpalphalangeal joints, the wrists, $10 \mathrm{~cm}$ distal to the lateral epicondyles, and $12 \mathrm{~cm}$ proximal to the lateral epicondyles, as per the American Cancer Society's treatment manual while the patient was sitting upright in a chair with the shoulder in 90-degree flexion (20). A 9-mm wide, bendable, and non-elastic tape with a sensitivity level $0.1 \mathrm{~cm}$ was used for in circumferential measurements. Measurements were made separately for both upper limbs and the difference was recorded in $\mathrm{cm}$.

Limb volumes were determined by using the circumferential measurements in the Frustum formula (truncated cone formula) (21). The difference between the volumes of two limbs was recorded in milliliters $(\mathrm{mL})$.

\section{Secondary outcomes: grip strength, upper extremity function and quality of life}

Grip strength was assessed using a Jamar hydraulic hand dynamometer (Sammons Preston Rolyan, Bolingbrook, IL) with readings in kilograms. Three consecutive measurements were done at 30-second intervals. The patients were instructed to squeeze the instrument with their maximum strength in the standard position (shoulder adduction, forearm in neutral position, elbow 90-degree flexion, wrist 0-30-degree extension, and 0-15degree ulnar deviation). The average score of the three trials was recorded (22).

The 11-item Quick-Disabilities of the Arm, Shoulder and Hand (Q-DASH) questionnaire, was used to determine the upper limb functional level on a 5-point Likert scale (with $1=$ "no difficulty" and 5= "greatest difficulty") and the total Q-DASH score (0 shows no disability, 100 shows the most severe level of disability) was calculated (23).

The Functional Assessment of Cancer
Therapy-Breast (FACT-B) was used for the assessment of quality of life (24). This scale has five subscales: physical well-being ( 7 items), social/family well-being ( 7 items), emotional well-being ( 6 items), functional well-being ( 7 items), and additional concerns (10 items) rated on a 5-point Likert scale (from 0 , not at all to 4 , very much) with higher scores indicating better quality of life.

\section{Statistical Analysis}

The Statistical Package for the Social Sciences (SPSS) software (Version 22.0, IBM Corp., Armonk, NY, USA) was used for statistical analysis. The descriptive statistics of the data are expressed as mean, standard deviation (SD) and median values for continuous variables, and counts and percentages for categorical variables. Chi-square test was used in the analysis of qualitative independent data. The Kolmogorov-Smirnov test was used to analyze the normality of distribution of quantitative variables. The Mann-Whitney-U test was used to compare the means between groups, and the Wilcoxon test was used to evaluate the parameters within the groups. A $p$ value of less than 0.05 was considered statistically significant.

\section{RESULTS}

\section{Participant Characteristics}

The demographic and clinical characteristics of the patients are summarized in Table 1. The final sample size was 36 (CDT, $n=19$; $\mathrm{KT}, \mathrm{n}=17$ ) as a result of the exclusion of 4 patients from the final analysis due to patients' refusal of treatment, adverse effects, and nonadherence (Fig. 2). Dropout patients were not included in the statistical analysis. Tape renewal was performed in only 3 patients $(17 \%)$ in KT group. There was no significant difference between the two groups in terms of demographic and clinical characteristics and initial evaluation of all assessments ( $p>0.05$ ) although BMI was just outside of a significant difference $(\mathrm{p}=0.052)$. 
TABLE 1

\section{BASELINE CHARACTERISTICS OF THE PATIENTS}

\begin{tabular}{|c|c|c|c|c|c|}
\hline \multirow{2}{*}{ Characteristics } & \multicolumn{2}{|l|}{$\begin{array}{l}\text { CDT group } \\
(n=19)\end{array}$} & \multicolumn{3}{|l|}{$\begin{array}{l}\text { KT group } \\
(\mathrm{n}=17)\end{array}$} \\
\hline & $\begin{array}{l}\text { Mean } \pm \text { SD or } n \\
\text { (\%) }\end{array}$ & Median & $\begin{array}{l}\text { Mean } \pm \text { SD or } n \\
\text { (\%) }\end{array}$ & Median & p \\
\hline Age (years) & $53.4 \pm 8.3$ & 54.0 & $53.7 \pm 8.6$ & 56.0 & 0.707 \\
\hline BMI $\left(\mathrm{kg} / \mathrm{m}^{2}\right)$ & $26.7 \pm 5.1$ & 25.5 & $29.7 \pm 4.4$ & 29.3 & 0.052 \\
\hline $\begin{array}{l}\text { Lymphedema duration } \\
\text { (years) }\end{array}$ & $2.6 \pm 1.6$ & 3.0 & $2.8 \pm 1.8$ & 2.0 & 0.616 \\
\hline Affected extremity, right/left & $14(73.7) / 5(26.3)$ & & $8(47.0) / 9(52.9)$ & & 0.171 \\
\hline $\begin{array}{l}\text { Lymphedema side, } \\
\text { dominant/non-dominant }\end{array}$ & $11(57.9) / 8(42.1)$ & & $7(41.2) / 10(58.8)$ & & 0.505 \\
\hline Pain, yes/no & $5(26.3) / 14(73.7)$ & & $8(47.0) / 9(52.9)$ & & 0.299 \\
\hline $\begin{array}{l}\text { Types of surgery } \\
\text { Breast conservation } \\
\text { Modified radical } \\
\text { mastectomy } \\
\end{array}$ & $\begin{array}{r}13(68.4) \\
6(31.6)\end{array}$ & & $\begin{array}{r}15(88.2) \\
2(11.8)\end{array}$ & & 0.236 \\
\hline $\begin{array}{l}\text { Dissected lymph nodes, } \\
<15 />15 \\
\text { Previous treatment for } \\
\text { lymphedema }\end{array}$ & $9(47.3) / 10(52.6)$ & & $4(23.5) / 13(76.4)$ & & 0.177 \\
\hline $\begin{array}{l}\mathrm{CDT}+\mathrm{PG} \\
\mathrm{CDT}+\mathrm{PG}+\mathrm{PC} \\
\mathrm{PG} \\
\text { None }\end{array}$ & $\begin{array}{l}7(36.8) \\
3(15.8) \\
1(5.3) \\
8(42.1)\end{array}$ & & $\begin{array}{l}8(47.1) \\
3(17.6) \\
1(5.9) \\
5(29.4)\end{array}$ & & 0.885 \\
\hline
\end{tabular}

SD, Standard Deviation; BMI, body mass index; CDT, complete decongestive therapy; KT, Kinesiology Taping; PG, pressure garment; PC, pneumatic compression. Means (SD) is given for continuous variables; $\mathbf{n}(\%)$ is given for categorical data. $p$ values for continuous variables were calculated using Mann-Whitney-U test; $p$ values for categorical data were calculated using Pearson chi-squared test or Fisher exact test

\section{Primary Outcomes}

There was a statistically significant decrease in limb circumference difference in both the CDT and KT groups at the end of treatment $(-4.8 \pm 3.0 ; \mathrm{p}<0.001,-2.3 \pm 2.0 ; \mathrm{p}<0.001$,

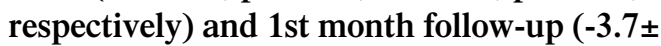
$4.2 ; \mathrm{p}=0.003,-2.4 \pm 2.0 ; \mathrm{p}=0.001$, respectively). When the groups were compared, the CDT group showed a statistically significant differ- ence after treatment $(\mathrm{p}=\mathbf{0 . 0 1 2})$ compared with the KT group, but this difference was not detected in the follow-up $(\mathrm{p}=0.121)($ Table 2 , Fig. 3).

There was a statistically significant decrease in lymphedema volume difference in both the CDT and KT groups at the end of treatment $(-121.6 \pm 74.8 ; \mathrm{p}<0.001,-60.7 \pm 46.1$; $\mathrm{p}=0.001$, respectively) and $1^{\text {st }}$ month follow-up $(-93.3 \pm 107.9 ; p=0.003,-65.0 \pm 53.8 ; p=0.001$, 


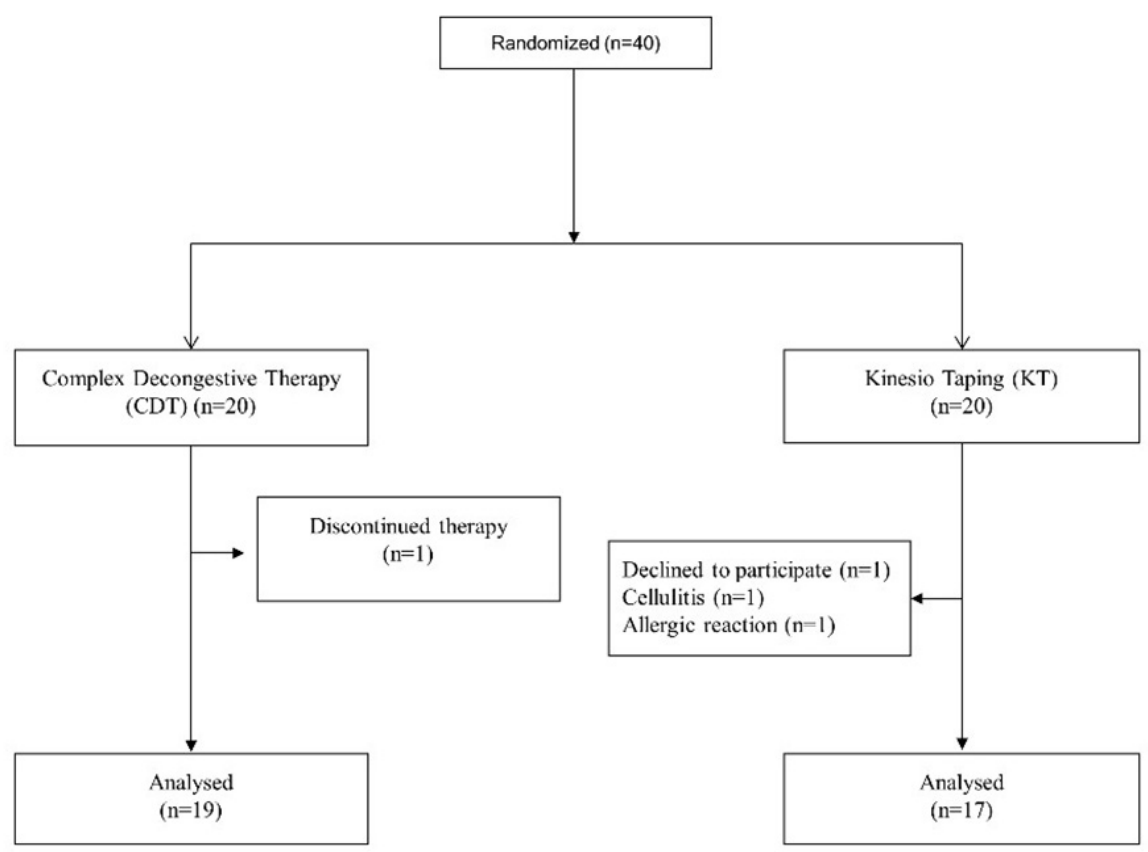

Fig. 2. Design and flow of patients through the study.

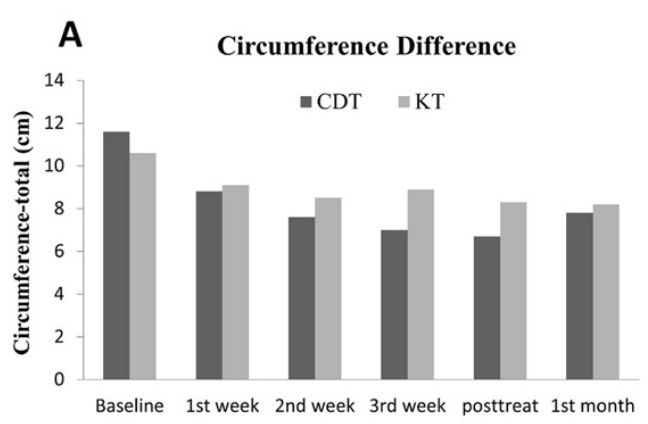

respectively). A significant difference was detected between the CDT and KT groups only at the end of the 4-week treatment $(\mathrm{p}=0$. 015) (Table 2, Fig. 3).

\section{Secondary Outcomes}

Significantly greater grip strength was detected in the CDT group only at the $1^{\text {st }}$ month follow-up $(1.1 \pm 1.5 ; \mathrm{p}=0.007)$, and in the KT group both at the end of treatment $(0.5 \pm$

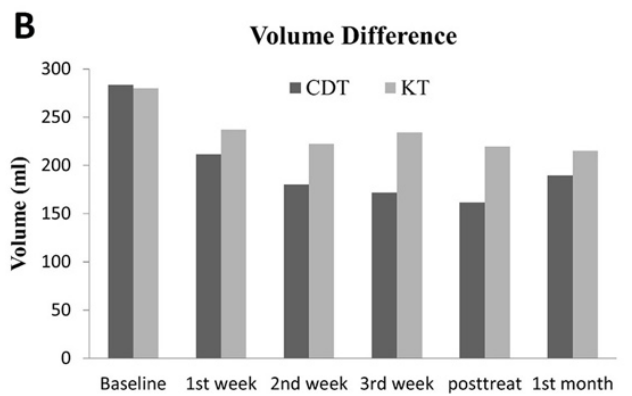

Fig. 3. Weekly upper limb circumference $(A)$ and volume differences $(B)$ in the CDT and KT groups. $0.8 ; \mathrm{p}=0.029$ ) and at the $1^{\text {st }}$ month follow-up $(0.7 \pm 0.7 ; p=0.004)$. There was no significant difference in terms of grip strength between the groups both at the end of treatment $(\mathrm{p}=0.707)$ and in the $\mathbf{1}^{\text {st }}$ month follow-up $(\mathrm{p}=0.754)($ Table 2).

Q-DASH scores were statistically significantly decreased in the CDT group at the end of treatment $(-2.8 \pm 5.3 ; \mathrm{p}=0.010)$, and in the KT group at both the end of treatment $(-3.2 \pm$ $5.9 ; \mathrm{p}=0.019)$ and at the $1^{\text {st }}$ month follow-up $(-3.8 \pm 11.3 ; p=0.031)$, but there was no signifi- 


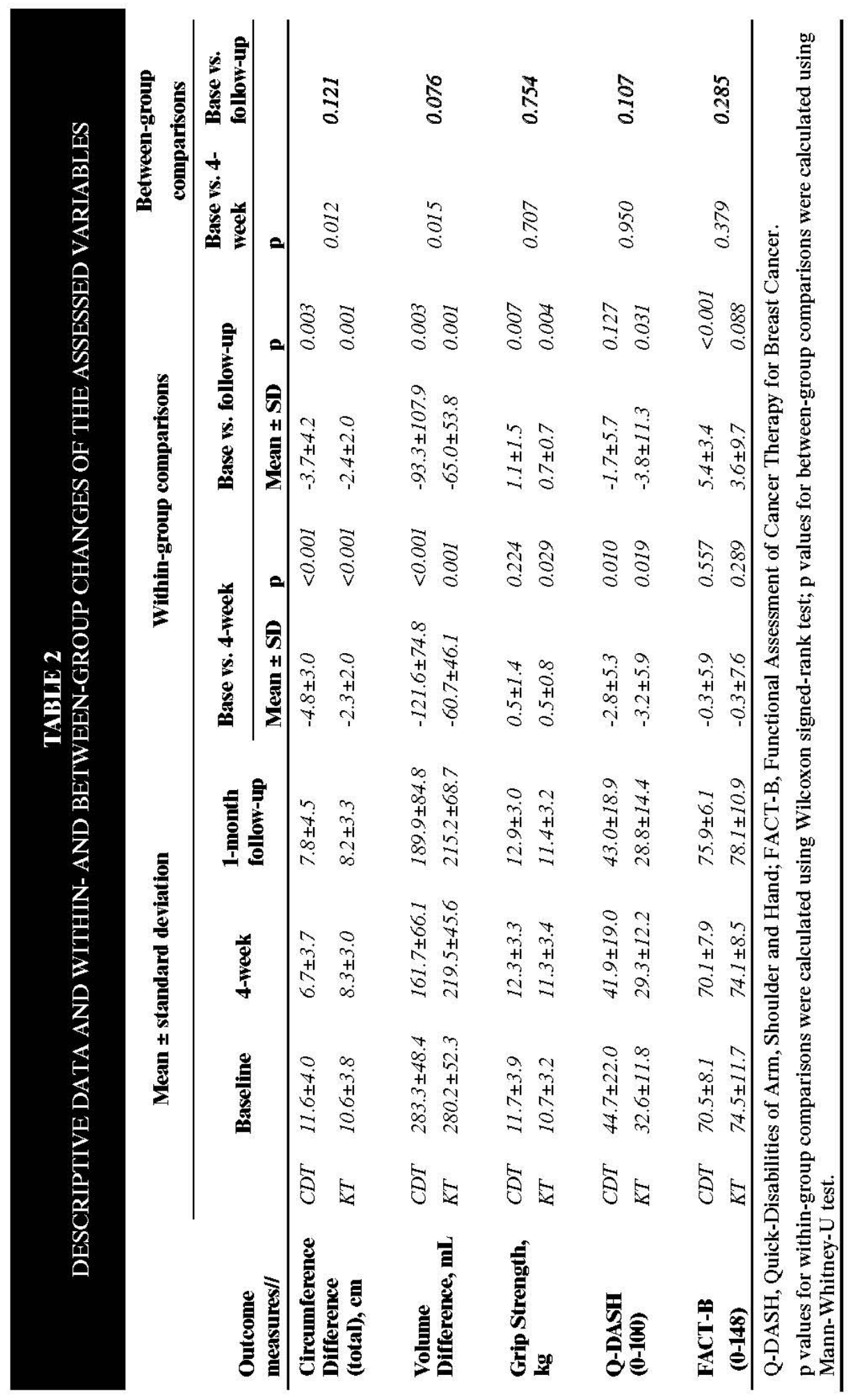


cant difference at the end of treatment and at follow-up ( $\mathrm{p}=0.950, \mathrm{p}=0.101$, respectively) between the groups (Table 2).

FACT-B scores, when evaluated within groups, showed a statistically significant increase in the CDT group at the 1st month follow-up (5.4 $\pm 3.4 ; p<0.001)$, however, in the comparison between the groups, but there was no significant difference in FACT-B scores after treatment and at follow-up compared with the beginning measures $(\mathrm{p}=0.379$, $\mathrm{p}=0.285$, respectively) (Table 2 ).

\section{DISCUSSION}

This study compared the effects of KT (with skin care and exercise) to CDT in treating patients with BCRL. Our study is the only study we are aware of from the literature that compares the intensive phase of CDT to KT. According to the results of our study, CDT and KT were not superior to each other in improving grip strength, function or quality of life, but CDT was superior to $\mathrm{KT}$ in reducing arm circumference and volume.

The findings of our study showed that $\mathrm{KT}$ was not superior to CDT in reducing edema in female patients with BCRL which is consistent with results of systematic reviews/ meta-analyses conducted by Kasawara et al (19) and Gatt et al (18). A study by Taradaj et al (25) randomized 70 patients with stage 2-3 BCRL to receive either KT with MLD and intermittent pneumatic compression (IPC) (the first group), quasi-KT (single-layered compression, low-pressure $<25 \mathrm{mmHg}$ ) with MLD and IPC (the second group), and multilayer bandaging with IPC and MLD (the third group). After four weeks of treatment, a statistically significant decrease was detected in the volume of the affected limb in all groups, but the third group which underwent bandaging showed a greater volume decrease than the other groups. It may be argued that the use of a KT application protocol similar to the one in our study (once a week, total of 4 sessions) may limit the effect of KT. However, in a randomized, controlled study, Smykla et al (26) divided 65 patients with stage 2-3 BCRL into $\mathrm{KT}$, quasi-KT, and multi-layer bandaging groups and administered skin care, 45-min IPC and 1-hour MLD for 4 weeks and found that the decrease in edema was greater in the bandaging group than in the taping groups.

In the studies by Taradaj et al and Smykla et al $(25,26)$, significant improvements were observed at the end of treatment even in normal quasi-KT group, indicating that MLD and IPC might also be effective in reducing lymphedema volume. In our study, unlike these other studies, the absence of MLD in the KT group may have contributed to the limb circumference and volume reduction in favor of CDT. Although it has also been noted by the ISL that the use of bandaging is an important part of conservative treatment, the actual role of MLD and IPC is still controversial $(12,27,28)$.

Tsai et al (15) compared the effects of modified CDT in which KT was performed instead of bandaging, and the effects of classic CDT including 4 weeks of skin care, bandaging, 30-minute MLD, 1-hour IPC, and a 20minute exercise program, on arm circumference, lymphedema volume, and quality of life in 41 patients with BCRL in their randomized controlled study. Contrary to the findings of our study, they found a statistically significant change in both arm circumference and lymphedema volume (water displacement) in the classic CDT group, and in only arm circumference in the KT group; however, there was no significant difference in all outcome variables between the groups. In that study, the patients used bandages for only an average of 7.8 hours per day and this limits comparisons to other studies. Although the volume of lymphedema significantly decreased, the use of bandages for a shorter time may have affected the results in favor of KT. Pekyavaş et al (29) randomized 45 patients with stage 2-3 lymphedema into 3 groups as CDT, CDT with KT used under the bandage, and KT without bandage. After 10 sessions of treatment, all patients were offered a self-lymphatic drainage, home exercise, and skin care as well as 
compression garments, and they were followed for one month. Although a statistically significant volume reduction was achieved in all groups at the end of treatment, it was reported that this effect continued until the 1st month follow-up in the second group receiving KT under the bandage, but no statistically significant difference was found between the groups. A potential reason for the long duration of the effect in the group receiving $\mathrm{KT}$ under the bandage can be explained by the fact that KT may increase the volume of lymphatic vessels in the dermis and it opens the area to superficial lymphatics of the skin.

In their meta-analysis including 5 randomized controlled trials, Gatt et al (18) observed that KT was not found to be more comfortable than bandaging, and a higher quality of life was achieved in patients receiving bandaging therapy. Similarly, the FACT-B scores statistically significantly increased in the CDT group after follow-up in our study. In addition, Pekyavaş et al (29) found that the SF-36 mental scores were higher in patients receiving bandaging, suggesting that bandaging might have a positive effect on mental functions of patients. However, in our study, both groups of patients had significant improvement in Q-DASH scores at the end of treatment, although it could be said that the statistically significant changes in the KT group rather than the CDT group were associated with the preservation of lymphedema volume in the KT group. Again, in our study, grip strength was not significantly increased in the CDT group at the end of treatment, but significantly increased in the KT group. The inverse correlation between grip strength and Q-DASH scores may have been due to the difference between doing a functional job and having a strong grip. Also, lymphedema symptoms (pain, limb ROM) play a greater role than the lymphedema volume in the increase of grip strength.

In their randomized controlled Tantawy et al (30) compared the effects of KT and pressure garments on limb circumference, Shoulder Pain and Disability Index (SPADI), hand grip strength, and quality of life. Sixty-six women with stage 2 and 3 BCRL who completed CDT phase I were divided into KT (2 times per week for 3 weeks) and pressure garment (20-60 mmHg, 15-18 hours a day for at least 3 weeks) groups. Arm circumference, shoulder pain, SPADI, hand grip strength, and quality of life were evaluated at baseline and at the end of treatment. Patients of KT group showed a significant improvement in all aspects whereas patients in the pressure garment group had no significant improvement except for significantly decreased limb circumference. This study is important in demonstrating that KT is effective in the maintenance phase, and future studies should investigate the effectiveness of KT in the maintenance phase rather than the intensive phase of CDT in the treatment of BCRL.

As far as we know, this study is the first randomized clinical trial to directly compare the efficacy of KT (with skin care and exercise) to the intensive phase of CDT in patients with BRCL. In addition to limb circumference measurements and lymphedema volume measurements, grip strength, upper limb function, and quality of life are among the strengths identified in our study. Another strength of the study was that KT and CDT were performed by experienced practitioners.

The first limitation of the study is the relatively small sample size. Another limitation is that all of the patients enrolled in the study had stage 2 lymphedema. Therefore, the results of the study cannot be generalized to mild and severe lymphedema (stages 1 and 3) treatment. Another limitation is that the measurement of limb volume was performed through indirect calculations using measurements of circumference, although this is a widely accepted measurement technique. Furthermore, the evaluation of outcome criteria by a blind researcher may have affected the results, and it may be argued that the KT group did not receive adequate intervention due to the administration of KT once a week. It is also not known whether patients increased their activity levels during treatment 
and follow-up and it cannot be ruled out that this may have affected the outcomes. Finally, we did not know if patients were practicing the prescribed home exercises and skin care because adherence to exercise and skin care was not monitored.

In conclusion, according to the results of this study, a total of 4 sessions of KT administered once a week was not significantly better or superior to CDT at the end of treatment and in the 1st month of follow-up in terms of improvement in limb circumference, lymphedema volume, grip strength, function or quality of life. It was, however, able to significantly reduce limb volume and circumference from baseline. At the end of the treatment, a statistically significant reduction in limb circumference and lymphedema volume was obtained in the CDT group compared with the KT group. These results showed that KT with skin care and exercise may not be supported as an alternative to MLD and bandaging in the intensive phase of classic CDT. However, due to poten-tial reduced costs and ease of application, effectiveness of KT and the optimal treatment protocol (number and frequency of sessions) should be tested in a larger number of patients with BRCL at different stages in future randomized controlled trials.

\section{CONFLICT OF INTEREST AND DISCLOSURE}

The authors declare no competing financial interests exist.

\section{REFERENCES}

1. Coleman, M, D Forman, H Bryant, et al: Cancer survival in Australia, Canada, Denmark, Norway, Sweden, and the UK, 1995-2007 (the International Cancer Benchmarking Partnership): An analysis of population-based cancer registry data. Lancet 377 (2011), 127-138.

2. Hayes, S, TD Sipio, S Rye, et al: Prevalence and prognostic significance of secondary lymphedema following breast cancer. Lymph. Res. Biol. 9 (2011), 135-141.

3. Forner-Cordero, I, J Muñoz-Langa, A Forner-Cordero, JM DeMiguel-Jimeno: Predictive factors of response to decongestive therapy in patients with breast-cancer-related lymphedema. Ann. Surg. Oncol. 17 (2010), 744-751.

4. Lee, TS, SL Kilbreath, KM Refshauge, et al: Prognosis of the upper limb following surgery and radiation for breast cancer. Breast Cancer Res. Treat. 10 (2008), 19-37.

5. McLaughlin, SA, S Bagaria, T Gibson, et al. Trends in risk reduction practices for the prevention of lymphedema in the first $\mathbf{1 2}$ months after breast cancer surgery. J. Am. Coll. Surgeons 216 (2013), 380-389.

6. DiSipio, T, S Rye, B Newman, S Hayes: Incidence of unilateral arm lymphoedema after breast cancer: A systematic review and metaanalysis. Lancet Oncol. 14 (2013), 500-515.

7. Hayes, SC, K Johansson, NL Stout, et al. Upper-body morbidity after breast cancer: Incidence and evidence for evaluation, prevention, and management within a prospective surveillance model of care. Cancer 118 (2012), 2237-2249.

8. Shaitelman, SF, KD Cromwell, JC Rasmussen, et al: Recent progress in the treatment and prevention of cancer-related lymphedema. Cancer 65 (2015), 55-81.

9. Finnane, A, M Janda, SC Hayes: Review of the evidence of lymphedema treatment effect. Am. J. Physic. Med. \& Rehab. 94 (2015), 483498.

10. Rogan S, J Taeymans, H Luginbuehl, et al: Therapy modalities to reduce lymphoedema in female breast cancer patients: A systematic review and meta-analysis. Breast Cancer Res. Treat. 159 (2016), 1-14.

11. Singh, B, T Disipio, J Peake, SC Hayes: Systematic review and meta-analysis of the effects of exercise for those with cancerrelated lymphedema. Arch. Phys. Med. Rehab. 97 (2016), 97:302-315. e13.

12. Executive Committee of the International Society of Lymphology. The diagnosis and treatment of peripheral lymphedema: 2020 Consensus Document of the International Society of Lymphology. Lymphology. 53 (2020), 3-19.

13. Smile, TD, R Tendulkar, G Schwarz, et al: A review of treatment for breast cancer-related 
lymphedema. Am. J. Clin. Oncol. 41 (2018), 178-190.

14. Jeffs, E, E Ream, C Taylor, et al: Clinical effectiveness of decongestive treatments on excess arm volume and patient-centered outcomes in women with early breast cancerrelated arm lymphedema: A systematic review. JBI Database System Rev. Implement. Rep. 16 (2018), 453.

15. Tsai, H-J, H-C Hung, J-L Yang, et al: Could Kinesio tape replace the bandage in decongestive lymphatic therapy for breastcancer-related lymphedema? A pilot study. Support Care Cancer 17 (2009), 1353.

16. Morris, D, D Jones, H Ryan, C Ryan: The clinical effects of Kinesio ${ }^{\circledR}$ Tex taping: A systematic review. Physiother. Theory Pract. 29 (2013), 259-270.

17. Aguilar-Ferrándiz, ME, AM Castro-Sánchez, GA Matarán-Peñarrocha, et al: Effects of kinesio taping on venous symptoms, bioelectrical activity of the gastrocnemius muscle, range of ankle motion, and quality of life in postmenopausal women with chronic venous insufficiency: A randomized controlled trial. Arch. Phys. Med. Rehabil. 94 (2013), 2315-2328.

18. Gatt, $M, S$ Willis, $S$ Leuschner: A metaanalysis of the effectiveness and safety of kinesiology taping in the management of cancer-related lymphoedema. Eur. J. Cancer Care 26 (2017), e12510.

19. Kasawara, KT, JMR Mapa, V Ferreira, et al: Effects of Kinesio Taping on breast cancerrelated lymphedema: A meta-analysis in clinical trials. Physiother. Theory Pract. 34 (2018), 34:337-345.

20. Harris, SR, KH Schmitz, KL Campbell, ML McNeely: Clinical practice guidelines for breast cancer rehabilitation: Syntheses of guideline recommendations and qualitative appraisals. Cancer 118 (2012), 2312-2324.

21. Sukul, DK, P Den Hoed, E Johannes, et al: Direct and indirect methods for the quantification of leg volume: Comparison between water displacement volumetry, the disk model method and the frustum sign model method, using the correlation coefficient and the limits of agreement. J. Biomed. Engineer. 15 (1993), 477-480.

22. Tredgett, M, T Davis: Rapid repeat testing of grip strength for detection of faked hand weakness. J. Hand Surgery 25 (2000), 372-375.
23. Beaton, DE, JN Katz, AH Fossel, et al: Measuring the wole or the parts?: Validity, reliability, and responsiveness of the disabilities of the arm, shoulder and hand outcome measure in different regions of the upper extremity. J. Hand Ther. 14 (2001), 128-142.

24. Coster, S, K Poole, LJ Fallowfield: The validation of a quality of life scale to assess the impact of arm morbidity in breast cancer patients post-operatively. Breast Cancer Res. Treat. 68 (2001), 273-282.

25. Taradaj, J, T Halski, J Rosinczuk, et al: The influence of Kinesiology Taping on the volume of lymphoedema and manual dexterity of the upper limb in women after breast cancer treatment. Eur. J. Cancer Care 25 (2016), 647-660.

26. Smykla, A, K Walewicz, R Trybulski, et al: Effect of kinesiology taping on breast cancerrelated lymphedema: A randomized singleblind controlled pilot study. Biomed. Res. Int. (2013), 2013:767106

27. Ezzo, J, E Manheimer, ML McNeely, et al: Manual lymphatic drainage for lymphedema following breast cancer treatment. Cochrane Database Sys. Rev. 5 (2015), CD003475.

28. Tran, K, C Argáez: Intermittent pneumatic compression devices for the management of lymphedema: A review of clinical effectiveness and guidelines. Ottawa $\mathrm{ON}$ : Canadian Agency for Drugs and Technologies in Health; 2017.

29. Pekyava, NÖ, VB Tunay, T Akbayrak, et al: Complex decongestive therapy and taping for patients with postmastectomy lymphedema: A randomized controlled study. Eur. J. Oncol. Nursing 18 (2014), 585-590.

30. Tantawy, SA, WK Abdelbasset, G Nambi, DM Kamel: Comparative study between the effects of kinesio taping and pressure garment on secondary upper extremity lymphedema and quality of life following mastectomy: A randomized controlled trial. Integr. Cancer Ther. 18 (2019), 1534735419847276.

\author{
Ceyhun Basoglu, MD \\ Istanbul Faculty of Medicine \\ Istanbul University \\ Çapa - Fatih / ISTANBUL 34093, Turkey \\ Tel.: +90 2124142000 \\ Fax : +90 2126358522 \\ E-mail: ceyhunbasoglu@gmail.com
}

